\title{
A Sit-to-Stand Training Robot and Its Performance Evaluation: Dynamic Analysis in Lower Limb Rehabilitation Activities*
}

\author{
Enguo CAO**, Yoshio INOUE**, Tao LIU** and Kyoko SHIBATA** \\ ** Department of Intelligent Mechanical Systems Engineering, Kochi University of Technology \\ 185 Miyanokuchi, Tosayamada-Cho, Kami-City, Kochi 782-8502, Japan \\ E-mail: 138001p@gs.kochi-tech.ac.jp
}

\begin{abstract}
In many countries in which the phenomenon of population aging is being experienced, motor function recovery activities have aroused much interest. In this paper, a sit-to-stand rehabilitation robot utilizing a double-rope system was developed, and the performance of the robot was evaluated by analyzing the dynamic parameters of human lower limbs. For the robot control program, an impedance control method with a training game was developed to increase the effectiveness and frequency of rehabilitation activities, and a calculation method was developed for evaluating the joint moments of hip, knee, and ankle. Test experiments were designed, and four subjects were requested to stand up from a chair with assistance from the rehabilitation robot. In the experiments, body segment rotational angles, trunk movement trajectories, rope tensile forces, ground reaction forces (GRF) and centers of pressure (COP) were measured by sensors, and the moments of ankle, knee and hip joint were real-time calculated using the sensor-measured data. The experiment results showed that the sit-to-stand rehabilitation robot with impedance control method could maintain the comfortable training postures of users, decrease the moments of limb joints, and enhance training effectiveness. Furthermore, the game control method could encourage collaboration between the brain and limbs, and allow for an increase in the frequency and intensity of rehabilitation activities.
\end{abstract}

Key words: Dynamic, Rehabilitation, Robot, Control, Joint Moment

\section{Introduction}

Under the trend of population aging in many countries, the physical degeneration of aged persons leads to higher risk of deterioration of lower limb motor function, and stroke or brain injury have become common afflictions resulting in an increasing number of hemiplegics ${ }^{(1)}(2)$. Meanwhile, sports injuries, traffic accidents, childhood diseases and life-style related diseases can also cause standing-up disability. A large proportion of those affected cannot recover completely under current medical conditions, and rehabilitation training has become one of the most workable methods of treatment in many situations. Therefore, motor function recovery activities have aroused widespread interest and the effectiveness of muscle strength training equipments has been greatly progressed in many countries. Furthermore, self-controlled training, which can assure frequency and intensity of rehabilitation training without need for an expert assistant, has become favored in many institutions ${ }^{(3)}$.

Currently, balance training, limb coordination training and standing style transfer

[DOI: 10.1299/jsdd.6.466]

Copyright $@ 2012$ by JSME 
systems have provided adequate rehabilitation for many patients, and experiments have validated the practicality of these training systems ${ }^{(4-7)}$. Moreover, exoskeleton robots or prosthetic limbs have played an important role in the rehabilitation field. In order to offer motive power these robots usually decide the amount of moment or force by sensing the intention of patients ${ }^{(8)(9)}$, thereby raising the athletic ability of a physically weak body. But these rehabilitation activities may not ensure that training is undertaken in a natural and comfortable trajectory for the patients; moreover inadequate trajectory training may lead the injured limb to develop an unnatural trajectory. Meanwhile, rehabilitation robots with prescribed movement pattern or interactive motion rehabilitation have been developed for naturalistic motion and body weight supported training ${ }^{(10-13)}$, and a gravity-balancing leg passive orthosis also performed well in trajectory training ${ }^{(14)}$. But these rehabilitation systems are not capable of intelligently interpreting human intentions; they may just support an injured limb of a patient without concentrating on retraining weak points that may lead to unobvious rehabilitation effects.

Several more practical types of rehabilitation robotic devices have proved effective in helping improve limb motor function, but they may be not suitable for self-controlled training. For example, a gait rehabilitation robot with upper and lower limb connections that allows patients to update their walking velocity on various terrains ${ }^{(15)}$, a powered lower limb orthoses for gait rehabilitation that allows practice starting, turning, stopping, and obstacle avoidance during walking over ground ${ }^{(16)}$, and a bio-responsive motion system that works as a gait simulator for recovery of motion ability of the lower extremities of stroke patients ${ }^{(17)}$. However, these robots are relatively complex and difficult to set up by patients on their own, so sometimes the training can be conducted only in hospitals with an expert. These characteristics may increase the time pressure and economic pressure on patients, as rehabilitation training is normally a long-term activity. Furthermore, there is now sufficient evidence that using a rehabilitation protocol involving motor imagery practice in conjunction with physical practice of goal-directed rehabilitation tasks leads to enhanced functional recovery of paralyzed limbs among stroke sufferers ${ }^{(18)}$. Therefore, rehabilitation training games that can activate collaboration between brain and limb are necessary for developing effective recovery processes. In these circumstances, development of a comfortable and effective rehabilitation system for self-training would be valuable, and dynamic analysis of human limbs for evaluating the effect and interactivity of the rehabilitation activities is essential.

In this research, a sit-to-stand rehabilitation robot based on a double-rope system was developed, and the performance of the robot was evaluated by human lower limb dynamics analysis. In the robot system, precise locations of the wearable parts are unnecessary and control commands can be inputted by the patients on their own, which improved the weak point of Ref. 15-17. An impedance control method was developed to both ensure patients' comfortable postures which improved the weak point of Ref. 4-9, and respect patients' moving intention which improved the weak point of Ref. 10-14. In addition, a game control method was developed to improve the coordination of brain and limbs, which achieved the advantage of Ref. 18. In the other hand, for evaluating the performance of the robot, dynamic parameters of human lower limb was analyzed. In the rehabilitation activities, human segments rotational angles, movement trajectories, ground reaction forces (GRF), centers of pressure (COP) and rope tensile forces were measured by a sensor system, after which the joint moments of ankle, knee and hip were real-time calculated in the control program.

\section{Materials and Methods}

\subsection{Sit-to-stand Training Robot System}

In this research, a double rope rehabilitation robot was developed for training the lower 
limb motor functions. The level of assisting force provided by the robot is adjusted according to users' intention while assuring the adequate movement trajectory of body trunk. As shown in Fig. 1 (a), the whole hardware system is controlled by a computer program system ${ }^{(19)}$, the front and back ropes are connected to two servo motors, and the tensile forces of the ropes are real-time measured by two high precision load cells. In rehabilitation training activities, the GRF and the COP are real-time measured by the GRF sensor, and the angular motions of trunk, thigh, and shank are real-time measured by three wearable motion sensors. The assist force is real-time controlled by the back rope. The back rope can decide whether to offer assist forces or just follow the movement, because the sensor system and the algorithm controller can recognize the intended movement of the user. The back rope moves up faster when the user owns his/her own strength to stand up, moves up slower when the user needs help, or even moves down at a safe speed if the user intends to sit down. This ability ensures the rehabilitation system can spend more time training the weak posture of the user, and increases the recovery effect of the system.

Meanwhile, the movement trajectory is real-time controlled by the front rope. As shown in Fig. 1 (b), paired lengths of front rope and back rope decide the movement trajectory of point $\mathrm{C}$, so while the length of back rope is being decided by the assist force, the adequate trajectory of point $\mathrm{C}$ can be controlled by adjusting the length of front rope in the control program. However, the desirable trajectories of different individuals have to be prepared in the control program. As a research result in our laboratory, there is a proportion relation between the desirable movement trajectory and the height of user. For example, when a health man stands up by himself wearing the tautly following ropes of robot, the movement trajectory can be measured by the sensor system. If the trajectories of $1600(\mathrm{~mm})$ and 1800 $(\mathrm{mm})$ heights were measured by the robot system, the trajectory of $1700(\mathrm{~mm})$ height can be calculated by averaging the two trajectories, and the lengths of front rope and back rope should be averaged separately. In this way, the trajectory of any height can be prepared in the control program.

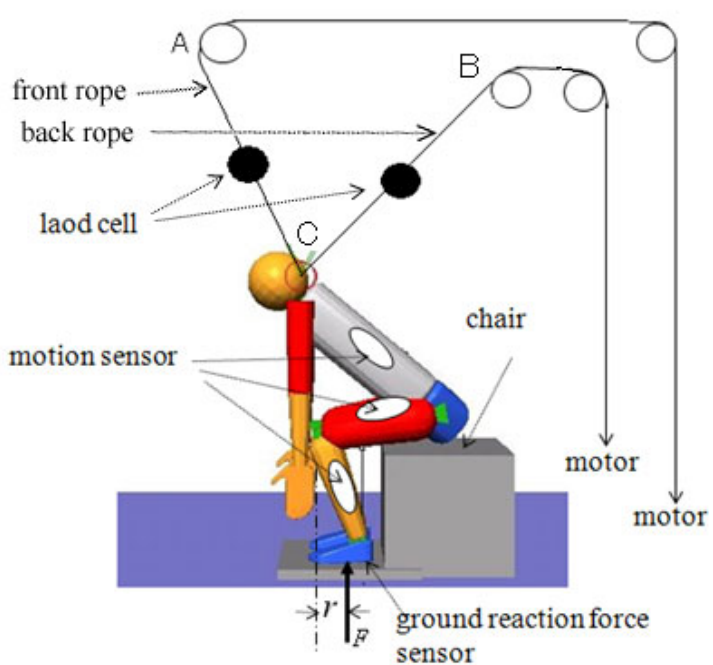

(a)

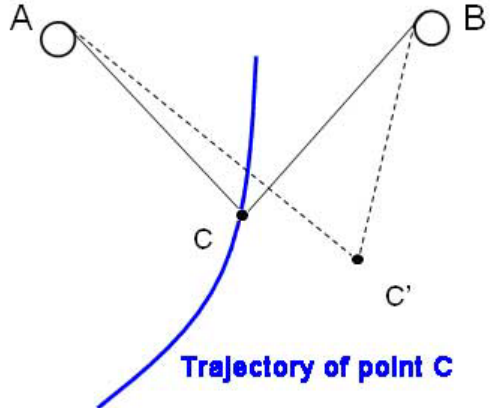

(b)

Fig. 1. Schematic diagram of the rehabilitation robot system.

\subsection{Sensor System}

The wearable motion sensors were constructed with inertial elements to measure the angular rotation of human shank, thigh, and trunk. As shown in Fig. 2 (a), the angular sensor recognizes the gravity direction of the accelerometer as a standard direction, and the 
changes of accelerometer direction can arouse voltage changes in the sensor, so the angle changes can be calculated out through measuring the voltage changes. This motion sensor system is quite inexpensive compared with conventional high-speed cameras system, and the measured data can be real-time utilized in the control program, furthermore the angular sensors can be easily fixed on body segments without precise location because the lower limbs are regarded as a rigid body system. A mini-type force plate was developed to measure the GRF and COP. As shown in Fig. 2 (b), four load cells are set at the location of $1,2,3$ and 4 of the force plate. Based on this structure, the total load (F) can be calculated by summation which is shown in Eq. (1), and the COP can be calculated with Eq. (2). The data measured by the force plate can be real-time utilized in the control program. In the robot system, the tensile forces of the two ropes are real-time measured by two load cells (LTZ-200KA), the length of the rope is real-time measured by a photoelectric encoder in the sever motors (MR-J2S), and the time is calculated per 0.1 (s) by the counter of the controller in the control program.

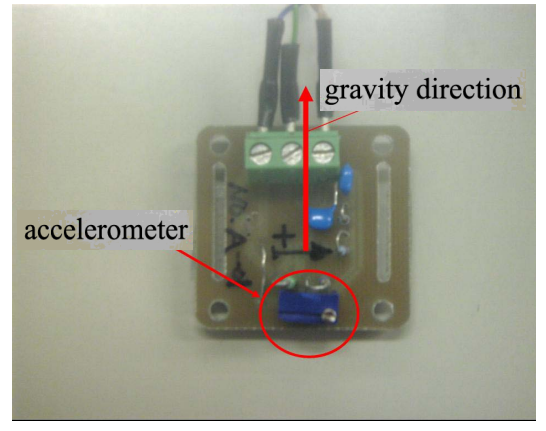

(a) Motion Sensor

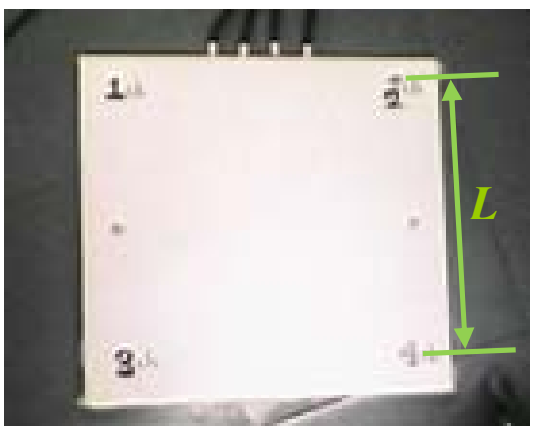

(b) Force Plate

Fig. 2. Schematic diagram of the structure of motion and GRF sensors.

$$
\begin{aligned}
& \mathrm{F}=F 1+F 2+F 3+F 4 \\
& \mathrm{COP}=L \cdot(F 3+F 4) / \mathrm{F}
\end{aligned}
$$

$L \quad$ - Distance from location 2 to location 4.

$F 1, F 2, F 3, F 4$ - Forces measured by load cell 1, 2, 3, 4 .

\subsection{Impedance Control and Game Control Methods}

An impedance control method was developed for the control program to improve the safety and robustness of the system. As shown in Fig. 3, before the user starts to stand up the ropes move up till taut in preparation for rehabilitation training, and when the user's intention to stand up is signaled the impedance control method starts immediately. As shown in equation (3) and Table 1, the back rope tensile force $(T)$ with the magnification coefficient of $b$ represents the movement intention of the user. Decreasing $T$ indicates that the user intends to stand up, while increasing $T$ indicates an intention to crouch down. For example, $10(\mathrm{~N})$ is a small value that can be easily achieved by patients, and $0.5(\mathrm{~s})$ is a reasonable minimum action time for patients. While the ropes are already taut in the preparation phase, $T$ decreasing by more than $10(\mathrm{~N})$ in $0.5(\mathrm{~s})$ indicates that the user intends to stand up and the impedance control method starts immediately. The displacement $(d)$, velocity $(v)$, and acceleration $(a)$ of point $\mathrm{C}$ on body trunk are real-time calculated from the lengths of ropes and the counter of controller in the control program, and the their changes are real-time impeded with the magnification coefficients of $k, c$, and $u$ respectively. Smaller values of $k, c$, and $u$ indicate that the user can move up or down flexibly, but larger values indicate that the displacement, velocity, and acceleration of ropes can not change rapidly and the system is therefore more safe and stable. Desirable values of $k, c$, and $u$ are 
decided by test experiments to assure that when the user stand up by himself tension on the rope can be maintained, and when the user drop down suddenly the back rope can move down at a safe speed.

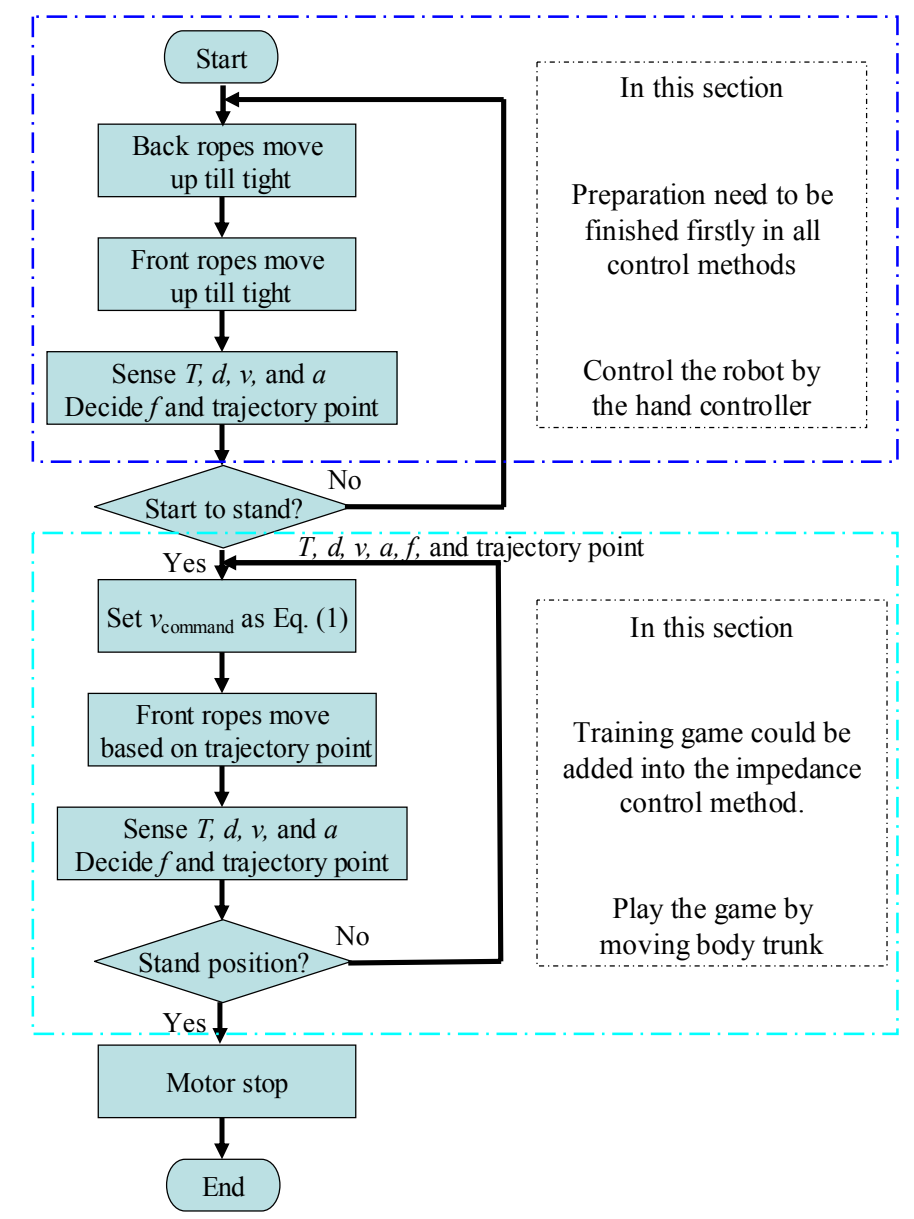

Fig. 3. A flow chart of impedance control method program, on which the game control method is based.

$$
v_{\text {input }}=(f-b \cdot T-k \cdot d-c \cdot v-u \cdot a) \cdot h
$$

Table I Definition of terms used in Equation 3

\begin{tabular}{cl}
\hline Terms & Definition \\
\hline$T$ & Back rope tensile force \\
$d$ & Displacement of point C on body trunk \\
$v$ & Velocity of point $\mathrm{C}$ on body trunk \\
$a$ & Acceleration of point $\mathrm{C}$ on body trunk \\
$b$ & Magnification coefficient of $T$ \\
$k$ & Magnification coefficient of $d$ \\
$c$ & Magnification coefficient of $v$ \\
$u$ & Magnification coefficient of $a$ \\
$f$ & Insurance parameter for rope tension and standing-up accomplishment \\
$h$ & Magnification coefficient of $v_{\text {input }}$ \\
$v_{\text {input }}$ & Velocity inputted into control commands.
\end{tabular}


Maintaining tension on the two ropes is the key requirement for the efficient working of the training system, so an insurance parameter $(f)$, which is always increasing in the impedance control method, is introduced to ensure accomplishment of the standing-up process and the tension on the ropes at all times. Considering the weight of conjunction jacket, the ropes are in danger of loosing when the tensile forces are lower than $50(\mathrm{~N})$, so $f$ increases quickly to maintain the rope tension while $T$ is lower than $50(\mathrm{~N})$. Should the user stay in a weak posture for more than $10(\mathrm{~s}), f$ increases slowly to assist the user's upward movement at $1 / 5$ normal standing-up speed. Here 10 (s) means that the user owns no sufficient strength to move up but only stay there, 1/5 normal standing-up speed means longer training time can be maintained on the weak postures. This control method avoids sudden collapse of the user, but does not hinder the user while he/she is training at a normal speed. Longer training times can therefore be assured if the user drops in a weak posture. As shown in Table 2, for the safety considerations, the motor stops immediately when rope length, rope tensile forces, or motion angles exceed their predetermined ranges, furthermore the rope velocity remains $-1(\mathrm{~m} / \mathrm{s})$ or $1(\mathrm{~m} / \mathrm{s})$ when $v_{\text {input }}$ exceed $-1(\mathrm{~m} / \mathrm{s})$ or $1(\mathrm{~m} / \mathrm{s})$ respectively. As the rope velocity is almost $1(\mathrm{~m} / \mathrm{s})$ when a health man stands up in the robot, so this value seems quite reasonable for the safe peak value in rehabilitation activities.

Table 2 Ranges of terms

\begin{tabular}{ll}
\hline Terms & Ranges \\
\hline Rope tensile forces & Each rope has the range from zero to user's weight. For safety considerations, the \\
& motors stop when exceeding. In the sitting posture, the included angle of the two ropes \\
& is about $60^{\circ}$, the maximum upward force of point $\mathrm{C}$ is about $173 \%$ of user's weight. \\
& In the standing posture, the included angle is about $120^{\circ}$, the maximum upward force \\
& of point $\mathrm{C}$ is about $100 \%$ of user's weight. \\
& Each rope has the range from $-1(\mathrm{~m} / \mathrm{s})$ to 1 (m/s). For safety considerations, the rope \\
& velocity remains -1 ( $/ \mathrm{s})$ or 1 (m/s) when exceeding. In the sitting posture, the \\
& maximum upward or downward velocity of point $\mathrm{C}$ is about 1.73 (m/s). In the standing \\
posture, the maximum upward or downward velocity of point $\mathrm{C}$ is about 1 (m/s). & Each rope has the range from the rope length of sitting posture to the rope length of \\
& standing posture, which is depends on user's height. For safety consideration, the \\
Rope lengths & motors stop when exceeding. \\
& The motion angles of trunk, thigh, and shank have the ranges from $30^{\circ}$ to $100^{\circ}$, from \\
& $0^{\circ}$ to $100^{\circ}$, and from $30^{\circ}$ to $100^{\circ}$ respectively. Test experiments have validated \\
that these ranges are sufficient for normal standing-up postures. For safety & consideration, the motors stop when exceeding. \\
Motion angles &
\end{tabular}

In the game control method, a vertical direction rebound game was introduced for encouraging collaboration between brain and body in rehabilitation activities. In the game screen, the user controls a vertical blue board moving up or down to rebound a white ball, and the white ball will hit a brick and rebound back. The user can control the up-down movement of the board by moving his body trunk up or down with the assistance of the robot, and in total it takes users about five minutes to finish the game. The game control method developed is based on the impedance control method, and additional control algorithms are developed to improve activation of the brain during the training program. In the game control method, $f$ is always increasing but only returns to 0 when $T$ decreases more than $50(\mathrm{~N})$ in $0.5(\mathrm{~s})$. Here $0.5(\mathrm{~s})$ is a reasonable minimum action time for patients, and $50(\mathrm{~N})$ means a partly motor ability of patients. The system therefore allows the user to squat down at a safe speed only after the user showed a partly standing-up ability. Therefore, users who can not offer any force are unsuitable for the game control method, and they should first do rehabilitation training using the impedance control method. 


\subsection{Calculation of Joint Moments}

Different types of sensors can measure force and movement of human body segments, but joint moments are difficult to directly measure by sensors as it relates to many other parameters of human body. However, joint moment is one of the most important parameters in evaluating the motor function of human limbs, and it can reflect accurately the effectiveness of rehabilitation activities ${ }^{(20)}$. Therefore, in this paper the joint moments of hip, knee, and ankle are calculated based on the data measured by the sensor system. As shown in Fig. 4 (a), the magnitude and location of $F$ can be acquired from GRF and COP data measured by the force plate. $\theta_{1}, \theta_{2}$, and $\theta_{3}$ can be acquired from the angle data measured by the three wearable motion sensors, $L_{1}, L_{2}$, and $L_{3}$ can be acquired by the lower limb lengths of users, and $m_{1}, m_{2}$, and $m_{3}$ can be estimated by the weight of users and the mass percentage law of human body, which is shown in Fig. 4 (b). Moreover, the parameters $p, q$, and $w$ are introduced to indicate the mass centre locations of foot, shank, and thigh respectively ${ }^{(20)}$. As all the data are real-time measured by the sensor system of the rehabilitation robot, the joint moments can be real-time calculated in the computer control program. Finally the joint moments $(M)$ of ankle, knee and hip can be represented by the equation (4), (5), and (6).

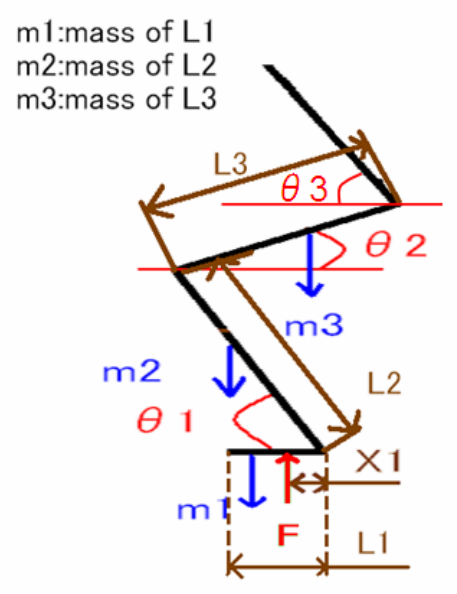

(a)

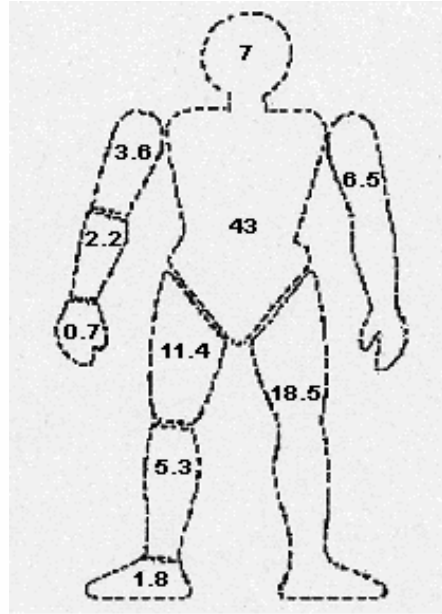

(b)

Fig. 4. A diagram illustrating dynamic and mass division in the human body.

$$
\begin{aligned}
M_{\text {ankle }} & =F x_{1}-p L_{1} m_{1} g \\
M_{\text {knee }}= & F\left[L_{2} \cos \left(\theta_{1}\right)-x_{1}\right]-q L_{2} \cos \left(\theta_{1}\right) m_{2} g \\
& -\left[L_{2} \cos \left(\theta_{1}\right)-p L_{1}\right] m_{1} g \\
M_{\text {hip }}=F & {\left[L_{3} \cos \left(\theta_{2}\right)-L_{2} \cos \left(\theta_{1}\right)+x_{1}\right]-w L_{3} \cos \left(\theta_{2}\right) m_{3} g } \\
& -\left[L_{3} \cos \left(\theta_{2}\right)-q L_{2} \cos \left(\theta_{1}\right)\right] m_{2} g \\
& -\left[L_{3} \cos \left(\theta_{2}\right)-L_{2} \cos \left(\theta_{1}\right)+p L_{1}\right] m_{1} g
\end{aligned}
$$

$$
\begin{array}{cl}
p & - \text { Parameter of mass centre locations of foot. } \\
q & - \text { Parameter of mass centre locations of shank. } \\
w & - \text { Parameter of mass centre locations of thigh. }
\end{array}
$$

\section{Sit-to-stand Rehabilitation Training Experiment}

\subsection{Experiment Method}

For analyzing the dynamic parameters of human lower limb and the effectiveness of the 


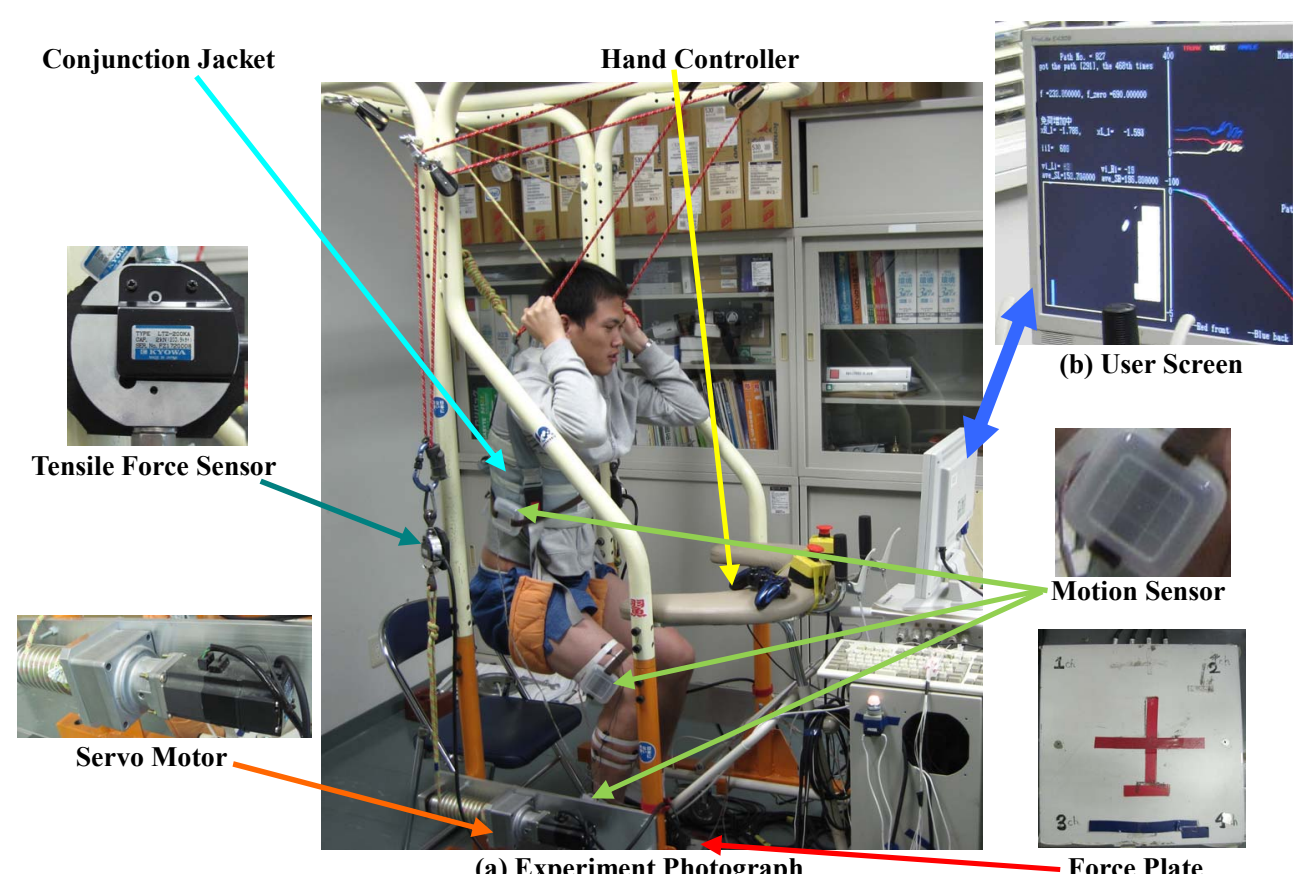

(a) Experiment Photograph

Force Plate

Fig. 5. Photograph of a subject in the rehabilitation experiment.

rehabilitation system, test experiments were designed for four subjects (age: $26 \pm 5$ years, height: $167 \pm 10 \mathrm{~cm}$, mass: $52 \pm 9 \mathrm{~kg}$ ) who have no history of musculoskeletal pathology or injury. As shown in Fig. 5 (a), the subjects were requested to stand up from a chair at self-selected speeds using, respectively, the self-standing method, the impedance control method and the game control method. In the start posture, the subject's elbow joints were in contact with the homologous knee joints, while in the terminal posture the subject's legs were straight. In the experiment the subjects were attached to the training robot by a conjunction jacket, and they were requested to always keep their feet on the force plate. The three wearable motion sensors were fixed by belts on the trunk, thigh, and shank respectively. Precision location of the motion sensors attached to limbs is unnecessary because all the limb segments are regarded as rigid segments. Moreover, the conjunction jacket and wearable sensors are easy to wear without expert assistance, and control commands can be inputted with a hand controller. While the subjects were standing up, the movement trajectories, rope tensile forces, COP, GRF, and angular motions of trunk, thigh, and shank were real-time measured, and the joint moments of hip, knee, and ankle were real-time calculated out in the control program. As shown in the user screen of Fig. 5 (b), control parameters, training game, joint moments, and movement trajectory were displayed in the upper left, lower left, upper right, and lower right area of the screen respectively. In the self-standing experiment the subjects stood up on their own while the ropes followed the body movements and remained taut. In the impedance control experiment the subjects hypothesized that their legs were injured and that they had not enough strength to stand up independently, in order to see whether the trainer system could assist them in completing the process of standing-up. And in the game control experiment, the subjects moved their body trunk up or down to control a blue board on a screen to move up or down, which he/she used to rebound a white ball, somewhat like a game of squash. Before the experiment, the objective and method of the experiment were explained to the subjects, and their written and oral consent to the experiment was obtained. This experiment had been pre-approved by the ethics committee of the Department of Intelligent Mechanical System Engineering, Kochi University of Technology. 


\subsection{Experimental Results}

As body parameters and individualities are different among different subjects, the experiment results were not in perfect accordance. However, similar tendencies were found. As shown in Fig. 6, photograph represented five postures in $0 \%, 30 \%, 50 \%, 70 \%$, and $100 \%$ of standing-up process (SP). To make the figures clear, the experiment results of three subjects using the impedance control method were shown from Figs. 7 to Fig. 11. The horizontal axis indicated one SP cycle, which is a posture series. In the control program, one posture can be recognized multiple times, which means one posture can be trained by longer time. As shown in Fig. 12, training times on different posture sections were summarized. Furthermore, different individuals were represented by different colors in all the figures.

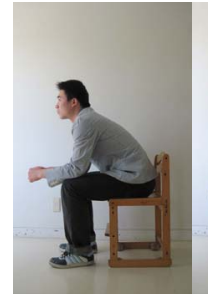

$0 \%$

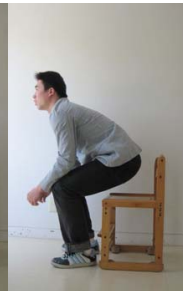

$30 \%$

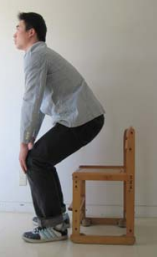

$50 \%$

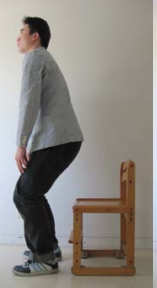

$70 \%$

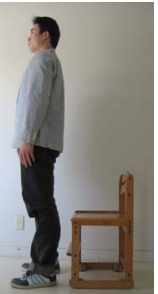

$100 \%$

Fig. 6. Photograph of five postures in $0 \%, 30 \%, 50 \%, 70 \%$, and $100 \%$ of standing-up process (SP).

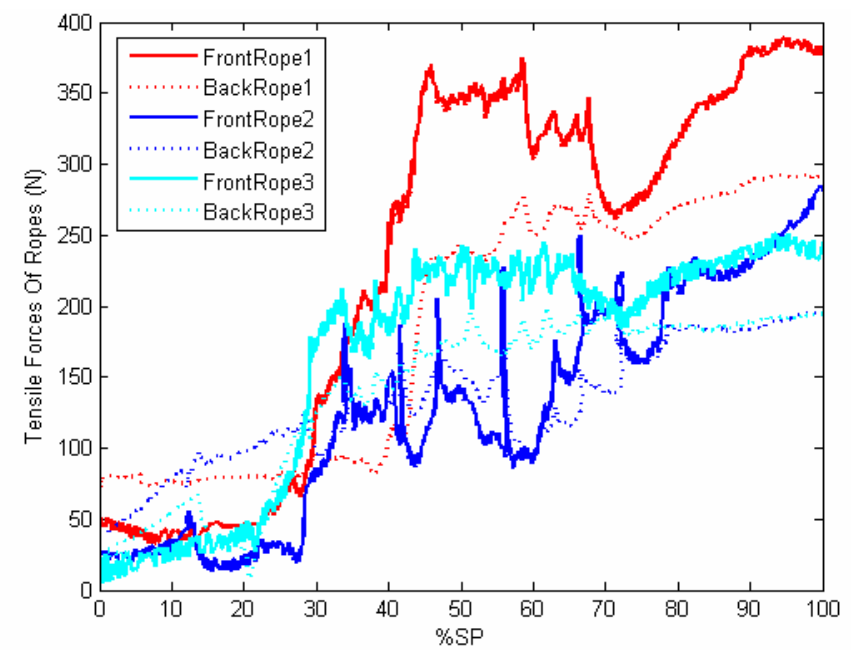

Fig. 7. Rope tensile forces of three subjects in impedance control method.

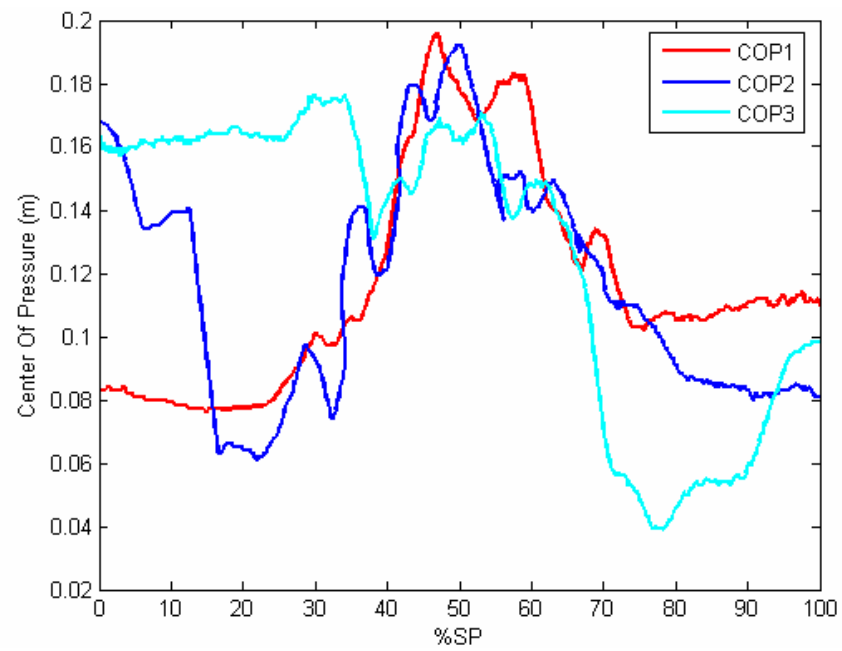

Fig. 8. COP of three subjects in impedance control method. 


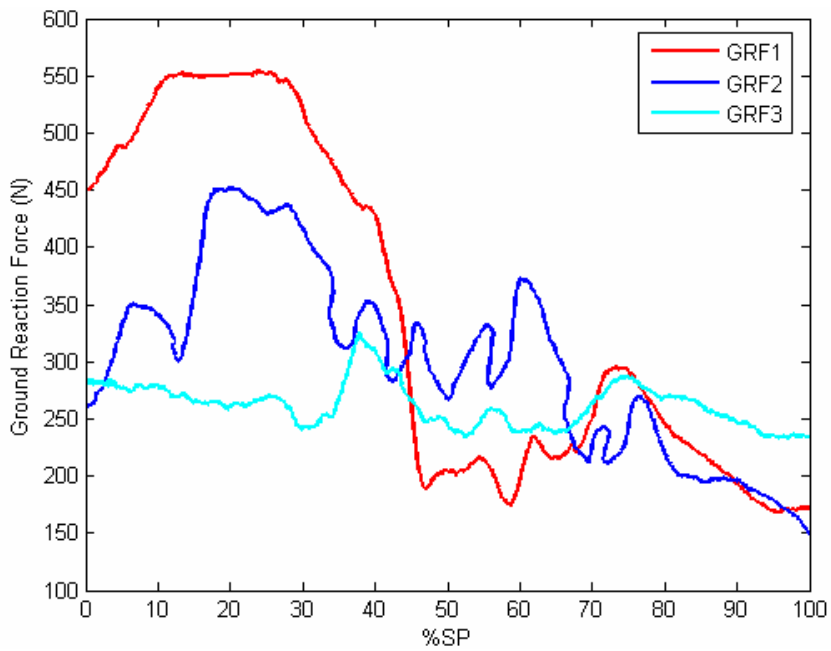

Fig.9. GRF of three subjects in impedance control method.

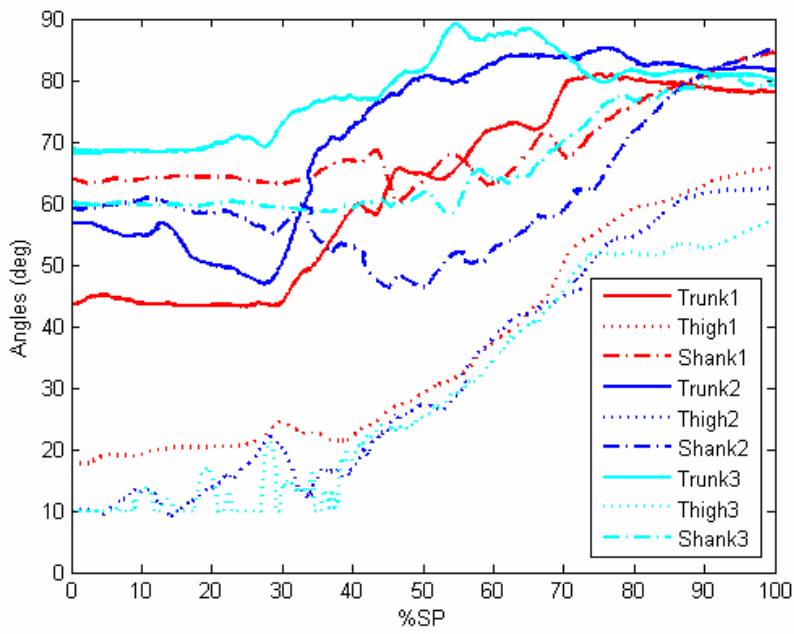

Fig. 10. Motion angles of shank, thigh, and trunk of three subjects in impedance control method.

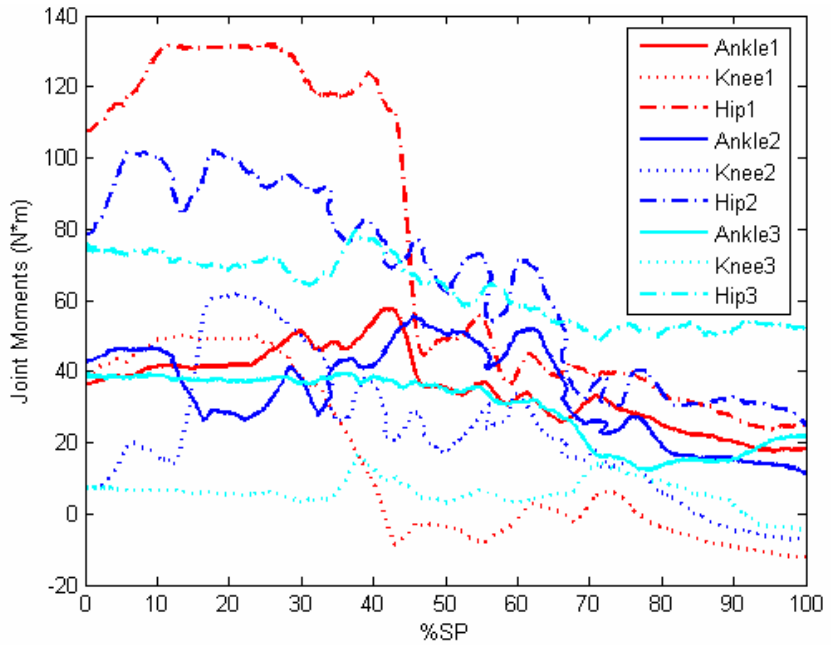

Fig. 11. Joint moments of ankle, knee, and hip of three subjects in impedance control method. 


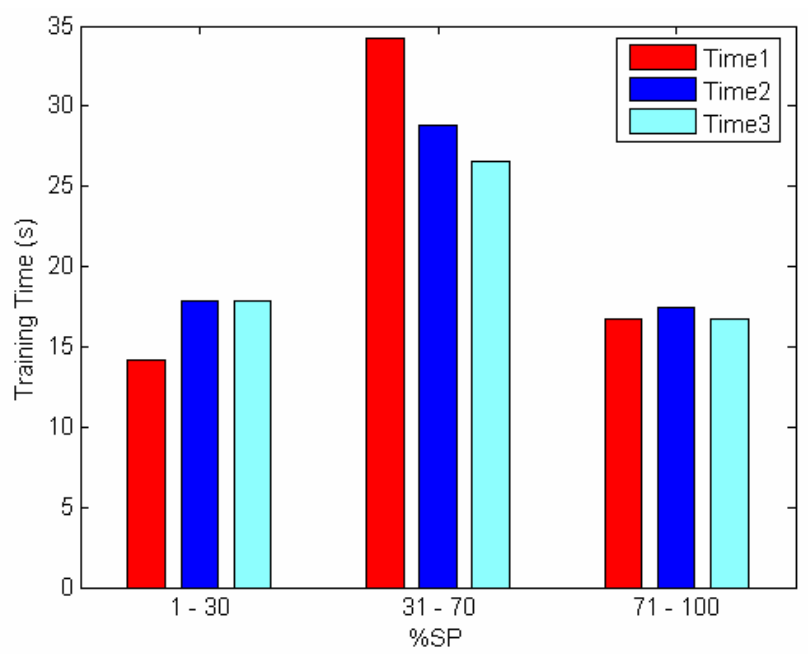

Fig. 12. Training times of different posture sections of three subjects in impedance control method.

To analyze the dynamic parameters of human lower limbs in different control regimes, the contrastive experiment results of the fourth subject using, respectively, the self-standing method, the impedance control method, and the game control method were shown from Fig. 13 to Fig. 18. To make the figures clear, data from different control methods were drawn with different colors.

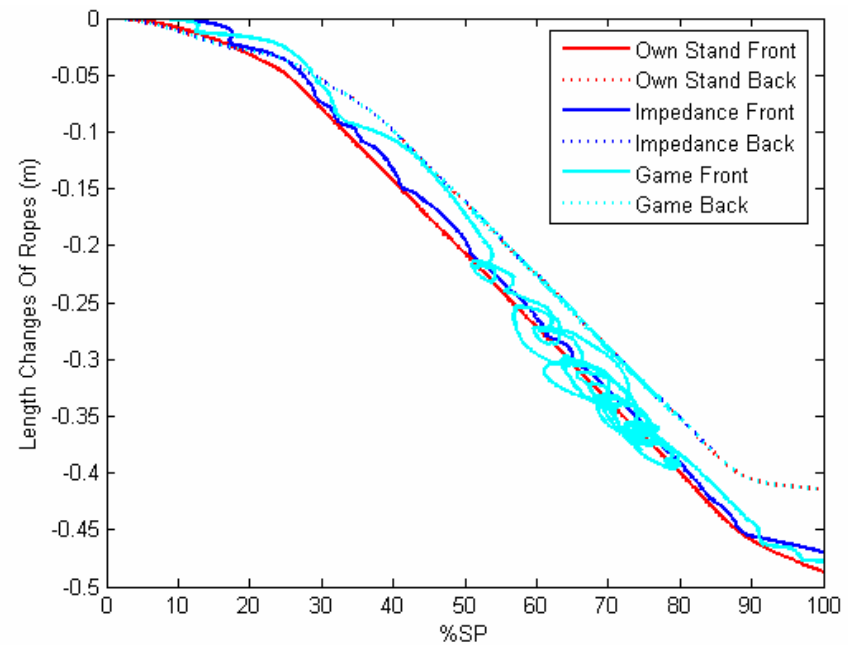

Fig. 13. Movement trajectories of the fourth subject recorded during three control methods.

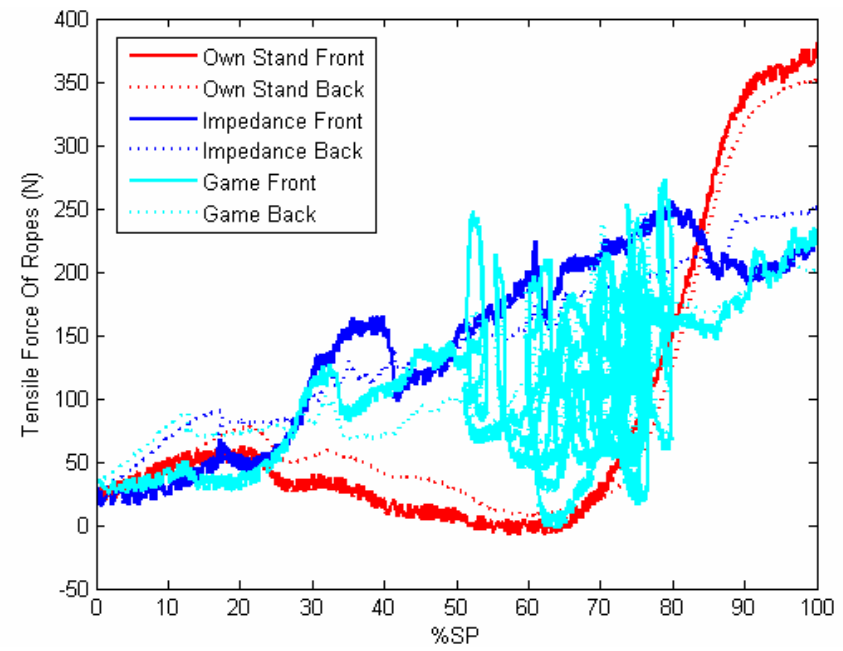

Fig. 14. Rope tensile forces of the fourth subject recorded during three control methods. 


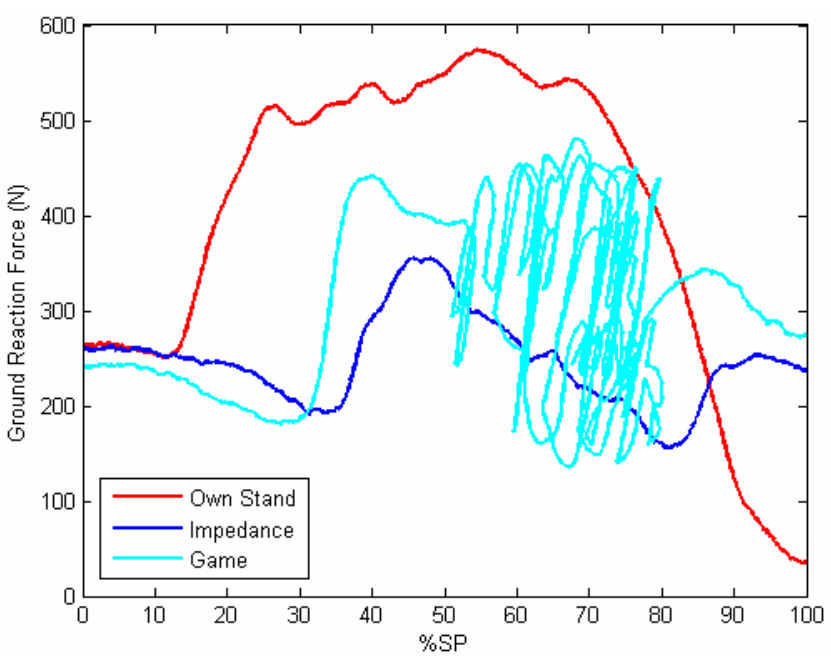

Fig. 15. GRF of the fourth subject recorded during three control methods.

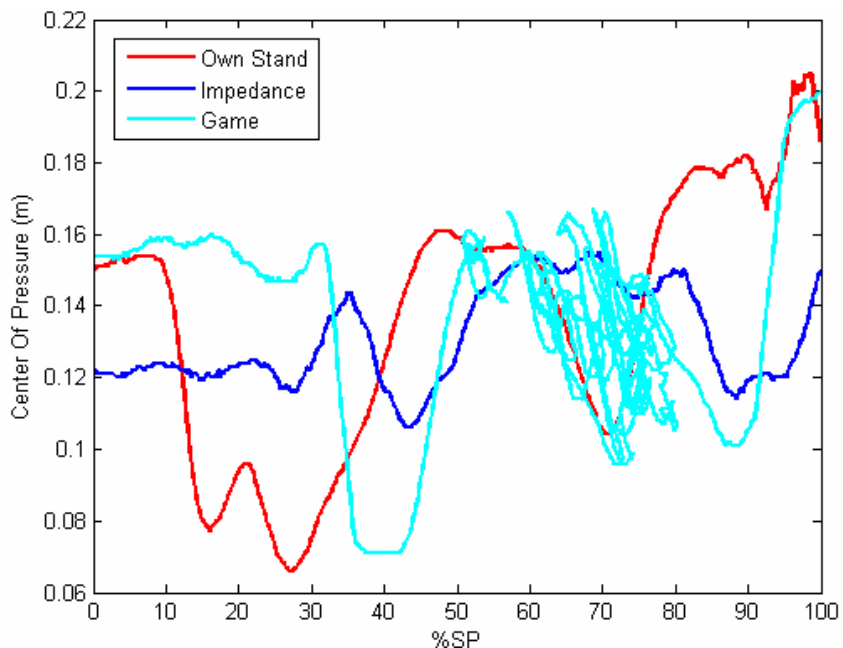

Fig. 16. COP of the fourth subject recorded during three control methods.

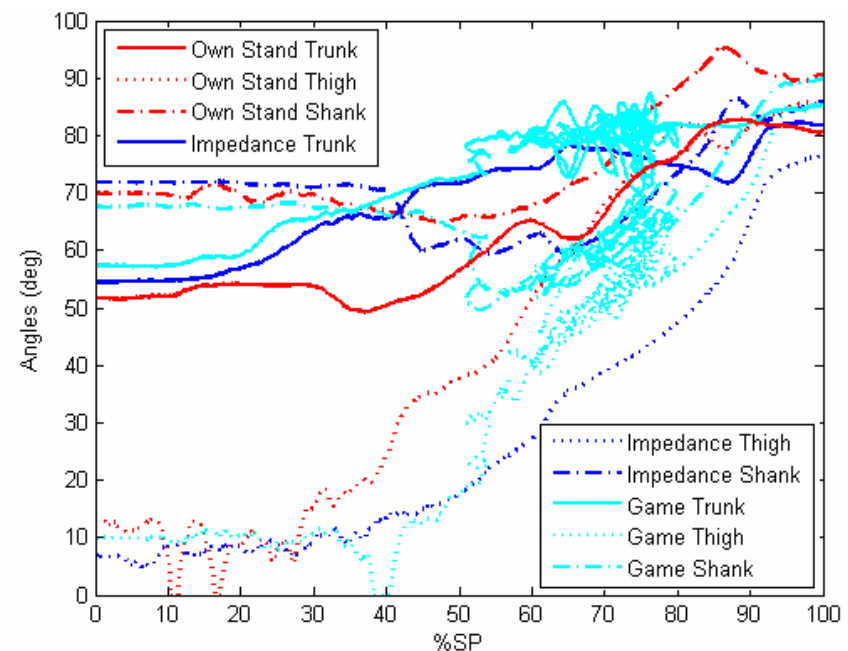

Fig. 17. Motion angles of shank, thigh, and trunk of the fourth subject recorded during three control methods. 


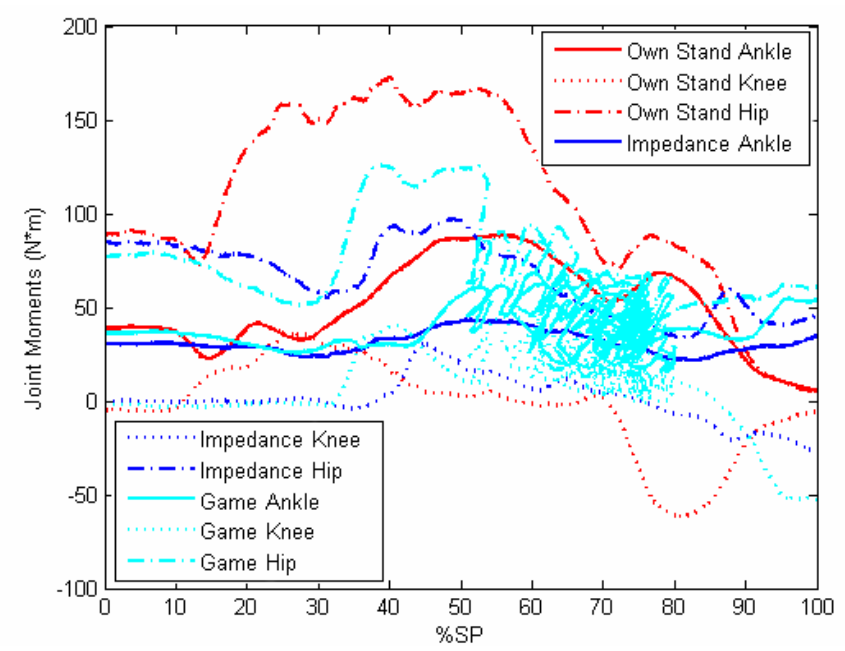

Fig. 18. Joint moments of ankle, knee, and hip of the fourth subject recorded during three control methods.

\section{Discussions}

The experiment results of three subjects in impedance control method were shown from Figs. 7 to Fig. 12. As shown in Fig. 7, from $30 \%$ to $70 \%$ of SP, the tensile forces of front ropes were higher than the back ropes. As shown in Fig.8, the COP showed a similar tendency to the tensile forces of the front ropes. The reason is that in our experiment we found that when a healthy man stands up from a chair the center of gravity of the human body always firstly moves forward and then moves back. Therefore in our training process, the front rope worked to keep a natural standing-up trajectory, so it offered a higher tensile force than the back rope in order to assure the forward movement of the gravity center. Whereas, the tensile forces of back rope increased gradually, meaning that the subjects stood up naturally and gradually in the SP. As shown in Fig.9, from $0 \%$ to $30 \%$ of SP, the GRF showed higher values when the subjects intended to stand up by their own, and after $30 \%$ of SP, the GRF decreased when the subjects felt more difficulty in standing and got more help from the robot. This is because the control program was designed to move up more gradually or even move down when subjects have insufficient strength, but to move up at a faster rate to maintain tension when subjects own sufficient strength to move up by their own. Therefore, vibratory values from $30 \%$ to $70 \%$ of SP indicate that the subjects were weak during this section and got more assistance from the robot. As shown in Fig.10, $\theta_{1}, \theta_{2}$, and $\theta_{3}$ grew continuously higher but only showed more vibrations in the weak posture section from $30 \%$ to $70 \%$ of SP. And in Fig. 11, the joint moments of hip, knee, and ankle vibrated in the weak posture section from $30 \%$ to $70 \%$ of SP, and showed higher values from $0 \%$ to $30 \%$ of SP when the subject intended to stand up on their own. In the experiment, the peak joint moment values of hip, knee, and ankle were, respectively; 132.1 $(\mathrm{N} \cdot \mathrm{m})$ occurring in the $26.1 \%$ of SP of subject $1,61.7(\mathrm{~N} \cdot \mathrm{m})$ occurring in the $21.0 \%$ of SP at subject 2, and $57.5(\mathrm{~N} \cdot \mathrm{m})$ occurring in the $41.9 \%$ of SP of subject 1. As shown in Fig. 12, the training time on weak posture section was longer than other posture sections. In the weak posture section from $31 \%$ to $70 \%$ of SP, the training times were $34.2(\mathrm{~s}), 28.8(\mathrm{~s})$, and 26.6 (s) on subject 1,2 , and 3 , so the training times could be calculated as $8.55(\mathrm{~s} / 10 \%)$, $7.20(\mathrm{~s} / 10 \%)$, and $6.65(\mathrm{~s} / 10 \%)$. From $1 \%$ to $30 \%$ of SP, the training times could be calculated as $3.55(\mathrm{~s} / 10 \%), 4.47(\mathrm{~s} / 10 \%)$, and $4.47(\mathrm{~s} / 10 \%)$. From $71 \%$ to $100 \%$ of SP, the training times could be calculated as $4.17(\mathrm{~s} / 10 \%), 4.35(\mathrm{~s} / 10 \%)$, and $4.17(\mathrm{~s} / 10 \%)$. Furthermore, all dynamic parameters vibrated up and down more often in the weak posture section than in other posture sections. Therefore, the robot system spent more time concentrating on training the weak posture section. 
Contrastive experiment results of the fourth subject for the three control methods were shown from Figs. 13 to 18. As shown in Fig. 13, the movement trajectories were represented by the changes in rope length. In the three methods, the movement trajectories of back rope were in general accordance because the trajectory of back rope was regarded as a standard movement in the SP. The trajectory of front rope in impedance control method showed an unobvious larger fluctuation than in the self-standing method. This is because while the subjects were standing up on their own in impedance control method, the tensile forces were less than $50(\mathrm{~N})$, so the back rope moved up faster than normal speed, and the front rope followed the saved corresponding trajectory points and showed a hysteresis. Moreover, in the game control method, the front rope would make hysteresis when the subjects stood up by themselves; and the front rope would make a cycle when the subjects squatted down by themselves to play the training game. Nevertheless, the collective trajectories of front rope were still mainly in agreement, which means the human body always moved through a comfortable trajectory in the rehabilitation activity. As shown in Fig. 14 both the tensile forces of back rope and front rope showed higher values in impedance control method and in game control method, and as shown in Fig. 15 and Fig. 16, the GRF and COP showed conspicuously higher values and higher range of variation in the self-standing method. This is because in the impedance control method an assist force was provided and it made the GRF and COP grew slowly and stably. In the game control method - although the values fluttered up and down while playing the training game - the values of GRF was still lower than in the self-standing method, meaning the assist forces in impedance control method and game control method could decrease the load on the human body. In Fig. 17, the variations of $\theta_{1}, \theta_{2}$, and $\theta_{3}$ showed similarities across the three experiment methods, and this was in accordance with the similarity in the variations of the movement trajectories. And as shown in Fig. 18, the three joint moments of hip, knee, and ankle were higher in self-standing method than the other two methods, and the values vibrated up and down in game control method, demonstrating that the impedance control method could decrease the joint moments effectively, and the game control method could stimulate both enthusiasm for training and frequency of training while the brain and body collaborate with each other. Furthermore, no matter how many times the human body moved up and down in the game control method, the peak values of the joint moments were still lower than the values in self-standing method, showing that the game control method was effective in assuring that rehabilitation activities were performed safely.

\section{Conclusions}

A sit-to-stand rehabilitation robot was developed, and human lower limb dynamic parameters were analyzed for evaluating the performance of the robot. The analysis results showed that the rehabilitation robot based on impedance control theory and game control method could ensure the safe accomplishment of sit-to-stand process, maintain a comfortable training posture by working through natural movement trajectories, decrease joint moments by providing assist forces on ropes, and enhance training effectiveness by concentrating on training the weak posture section of users. Furthermore, the robot under the game control method could activate the collaboration of brain and limb, and increase the frequency and intensity of rehabilitation activities.

\section{Acknowledgement}

The authors wish to acknowledge the support of the volunteer subjects of Robotics and Dynamics Research Lab in Kochi University of Technology. 


\section{References}

(1) Fent, T., Counting (On) An Aging Population, IEEE Computing in Science \& Engineering, Vol.8, No.6 (2006), pp. 88-96.

(2) Mann, W.C., The Aging Population and Its Needs, IEEE Pervasive Computing, Vol.3, No.2 (2004), pp. 12-14.

(3) Van Exel, N. J. A., Koopmanschap, M. A., Scholte op Reimer, W., Niessen, L. W., and Huijsman, R., Cost-Effectiveness of Integrated Stroke Services, QJM-J. Assoc. Physicians, Vol.98, No.6 (2005), pp. 415-425.

(4) Jaime, R. P., Matjac`ic', Z., and Hunt, K. J., Paraplegic Standing Supported by FES-Controlled Ankle Stiffness, IEEE Trans. on Neural Systems and Rehabilitation Engineering, Vol.10, No.4 (2002), pp. 239-248.

(5) Peshkin, M., Brown, D. A., Santos-Munné, J. J., Makhlin, A., Lewis, E., Colgate, J. E., Patton, J., and Schwandt, D., 2005, KineAssist: A Robotic Overground Gait and Balance Training Device, Proc. of IEEE 9th Intl. Conf. on Rehabilitation Robotics, Chicago. USA (2005-7), pp. 241-246.

(6) Mori, Y., Okada, J., and Takayama, K., Development of a Standing Style Transfer System 'ABLE' for Disabled Lower Limbs, IEEE /ASME Trans. on Mechatronics, Vol.11, No.4 (2006), pp. 372-380.

(7) Vallery, H., Ekkelenkamp, R., Buss, M., and Kooij, H., Complementary Limb Motion Estimation based on Interjoint Coordination: Experimental Evaluation, Proc. of IEEE 10th Intl. Conf. on Rehabilitation Robotics, Noordwijk. Netherlands (2007-6), pp. 798-803.

(8) Tsukahara, A., Hasegawa, Y., and Sankai, Y., Standing-Up Motion Support for Paraplegic Patient with Robot Suit HAL, IEEE 11th Int. Conf. on Rehabilitation Robotics, Kyoto. Japan (2009-6), pp. 211-217.

(9) Riener, R., Lünenburger, L., Jezernik, S., Anderschitz, M., Colombo, G., and Dietz, V., Patient-Cooperative Strategies for Robot-Aided Treadmill Training: First Experimental Results, IEEE Trans. on Neural Systems and Rehabilitation Engineering, Vol.13, No.3 (2005), pp. 380-394.

(10) Aoyagi, D., Ichinose, W. E., Harkema, S. J., Reinkensmeyer, D. J., and Bobrow, J. E., A Robot and Control Algorithm That Can Synchronously Assist in Naturalistic Motion During Body-Weight-Supported Gait Training Following Neurologic Injury, IEEE Trans. on Neural Systems and Rehabilitation Engineering, Vol.15, No.3 (2007), pp. 387-400.

(11) Kuzelicki, J., Kamnik, R., Burger, H., and Bajd, T., Robot Assisted Standing-Up in Persons with Lower Limb Prostheses, Proc. of 23rd IEEE/EMBS Annual Conf. on Engineering in Medicine and Biology Society, Vol.2 (2001), pp. 1332-1335.

(12) Veneman, J. F., Kruidhof, R., Hekman, E. E. G., Ekkelenkamp, R., Asseldonk, E. H. F. V., and Kooij, H., Design and Evaluation of the LOPES Exoskeleton Robot for Interactive Gait Rehabilitation, IEEE Trans. on Neural Systems and Rehabilitation Engineering, Vol.15, No.3 (2007), pp. 379-386

(13) Neckel, N. D., Nichols, D., and Hidler, J. M., Joint Moments Exhibited by Chronic Stroke Volunteers While Walking with a Prescribed Physiological Gait Pattern, Proc. of IEEE 10th Intl. Conf. on Rehabilitation Robotics, Noordwijk. Netherlands (2007-6), pp. 771-775.

(14) Banala, S. K., Agrawal, S. K., Fattah, A., Krishnamoorthy, V., Hsu, W. L., Scholz, J., and Rudolph, K., Gravity-Balancing Leg Orthosis and Its Performance Evaluation, IEEE Trans. on Robotics, Vol.22, No.6 (2006), pp. 1228-1239.

(15) Yoon, J., Novandy, B., Yoon, C. H., and Park, K. J., A 6-DOF Gait Rehabilitation Robot With Upper and Lower Limb Connections That Allows Walking Velocity Updates on Various Terrains, IEEE/ASME Trans. on Mechatronics, Vol.15, No.2 (2010), pp. 201-215. 
(16) Ferris, D. P., Sawicki, G. S., and Domingo, A., Powered lower limb orthoses for gait rehabilitation, Top Spinal Cord Inj Rehabil., Vol.11, No.2 (2005), pp. 34-49.

(17) Hirata, R., Sakaki, T., Okada, S., Nakamoto, Z., Hiraki, N., Okajima, Y., Uchida, S., Tomita, Y., and Horiuchi, T., BRMS:Bio-Resposive Motion System (Rehabilitation System for Stroke Patients), Proc. of IEEE/RSJ Intl. Conf. on Intelligent Robots and Systems EPFL, Lausanne. Switzerland, Vol.2 (2002), pp. 1344-1348.

(18) Prasad, G., Herman, P., Coyle, D., McDonough, S., and Crosbie, J., Using Motor Imagery Based Brain-Computer Interface for Post-stroke Rehabilitation, Proceedings of the 4th International IEEE EMBS Conference on Neural Engineering, Antalya. Turkey (2009-5), pp. 258-262.

(19) Urban, M., and Bajcsy, P., Fusion of Voice, Gesture, and Human-Computer Interface Controls for Remotely Operated Robot, 7th International Conference on Information Fusion (FUSION), Vol.2 (2005-7), pp. 1644-1651.

(20) Bando, N., Horibe, S., Yamada, H., Morita, H., and Tanaka, K., Solid Body Link Model for the Motion of Standing Up and Capability of Evaluation of Joint Moment, Research Report of Life Technology Research Centre, Gifu Ken. Japan, No.2 (2006), pp. 30-37. 